\title{
Long-term stability plastic scintillation for LHAASO-KM2A
}

\author{
Jing Zhao \\ IHEP, China \\ E-mail: jzhao@ihep.ac.cn \\ Hongkui Lv* \\ IHEP, China \\ Chao Hou on behalf of the LHAASO Collaboration \\ IHEP, China
}

In order to study the long-term stability of plastic scintillator (PS) for LHAASO-KM2A, thermal aging tests of materials (BC-408, ND-100) were made. A forecast of tile operational time was made for normal conditions of usage (20\% reduction of light output). The plastic scintillator operational time can be estimated and its lifetime is determined from an Arrhenius plot.

35th International Cosmic Ray Conference - ICRC2017

10-20 July, 2017

Bexco, Busan, Korea

${ }^{*}$ Speaker. 


\section{Introduction}

The Large High Altitude Air Shower Observation(LHAASO)[1] is a proposed cosmic ray experiment with highest sensitivity $\left(1 \% I_{\text {crab }}\right)$ above $30 \mathrm{TeV}$ and the highest gamma ray all sky survey power, which will be build in Haizi Mountain, Sichuan Province of China, $4400 \mathrm{~m}$ a.s.l. It consists of an extensive air shower array covering $1 \mathrm{~km}^{2}$ (KM2A), three water Cherenkov detector arrays (WCDAs) and a wide field of view Cherekov telescope array(WFCTA). KM2A is focused on discovering the Galactic cosmic ray sources, searching for galactic gamma ray sources above $30 \mathrm{TeV}$ in the northern sky and measuring primary cosmic rays in the energy range of $10 \mathrm{TeV}$ to $100 \mathrm{PeV}$ [2]. It consists of 5195 electromagnetic particle detectors (EDs) and 1171 muon detectors (MDs).

ED is designed to detect the particle density and arrival times of secondary particles in Extensive Air Shower (EAS). The plastic scintillator is chosen as the main detection medium because of its high detection efficiency to charged particles, quick response time, good stability and low cost. The active area for a unit ED is $1 \mathrm{~m}^{2}$ and the time resolution should be better than 2 ns to reach a good angular resolution and high sensitivity for the whole array. The detection efficiency for charged particles should be better than 95\%. A $5 \mathrm{~mm}$ thick layer of lead plate is used to convert $\gamma$ into electron positron pairs to increase detection efficiency for secondary $\gamma$ and absorb charged particles with low energy to improve the angular resolution[3]. To reach the energy range of 10 $\mathrm{TeV}$ to $100 \mathrm{PeV}$, light output of MIP particle should $>20$ photoelectrons, the dynamic range of ED should cover $1-10000$ particles $/ m^{2}$.

Another important requirement of ED detector to PS is: PS's reduction on 20\% must occur not earlier than 10 years when it works at LHAASO site (the annual average temperature is $0^{\circ} \mathrm{C}$ ). It is important to develop a prognostic tool for sample stability. In this work the long-term stability of PS was experimentally studied for ED detector. To predict the PS operational time, the well-known principle of temperature time superposition is used [4]. This principle establishes the equivalency of temperature influence and its duration. Using it, accelerated aging was conducted in iso-thermal conditions under different temperatures that are usually higher than the operational temperatures. An accelerated thermal aging tests of scintillator at temperature of $60^{\circ} \mathrm{C}, 70^{\circ} \mathrm{C}$ and $80^{\circ} \mathrm{C}$ are conducted in this paper.

\section{Experimental method}

Environmental aging is a basic parameter characterizing long time stability even without the effect of radiation. Thermooxidative processes resulting in the creation of peroxides which absorb in the region of the scintillation radiation[5] Mechanical degradation due to chemical stress crazing which causes surface cracks that disrupt transmission. It is known that tile surface can 'craze'develop micro-cracks which rapidly destroys the capability of PS to transmit light by total internal reflection[6]. In all cases the light output decreases. In developing an accelerated aging test it is important to achieve the maximum likeness to the natural degradation processes. Those aging mechanisms lead to changes in the scintillation efficiency which in any realistic case is due to one or at most a few parameters, like temperature, humidity, water immersion. These parameters 
determine the practical usefulness of any scintillator composition. The influence of temperature for long times on the light output had been studied in this paper.

Comparative measurements were carried out on PS of the BC-408 (Saint-Gobain) and ND-100 (Nuvia), which are both polystyrene base. Those PS tiles were cut into $250 \mathrm{~mm} \times 250 \mathrm{~mm}$ and 20 mm thick with 8 grooves on the surface, 4 Wavelength Shift Fibers (WLS, 2.7m length, BCF-92) are passed through the scintillator and re-entered in an alternate groove. Each tile is wrapped with Tyvek(1082D) to increase photon collection efficiency (as shown in Figure 1). The signal from the PMT (XP2012B) connected to the WLS fiber was digitized using a VME charge ADC module and a TDC module, and readout with a PC. The light output of those two kinds of PS was measured. After dozens test of WLS fibers penetrate PS tiles, the connection of WLS fibers with PMT, the system error for this experiment is about $2.2 \%$.

The target PS tile sandwiched with the WLS fibers were placed between two trigger scintillation counters, each of which is $250 \mathrm{~mm}$ square and $20 \mathrm{~mm}$ thick. The spacing of two trigger counters is $100 \mathrm{~mm}$. A trigger signal was generated when both of the two trigger counters were hit by a cosmic-ray muon.

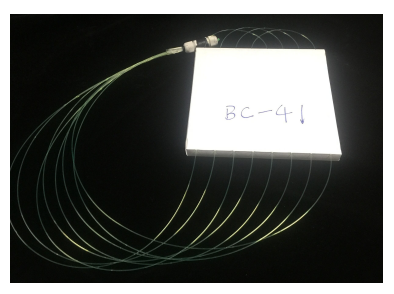

Figure 1: The PS tile with 4 WLS fibers.

\section{Experimental results}

Figure 2 gives the time dependence of the light output ratio of $60^{\circ} \mathrm{C}, 70^{\circ} \mathrm{C}$ and $80^{\circ} \mathrm{C}$. The aging plot can be fit by one exponential function:

$$
R=e^{\left(\frac{-t}{\tau}\right)}
$$

At $80^{\circ} \mathrm{C}$ heating, those tiles were heated more than 360 hours. The surface of BC-408 had lots of micro-cracks on the surface, these cracks were 5-10 $\mathrm{mm}^{-2}$ density but ND-100 has no change on the surface. After $30 \%$ reductions, these two kinds of PS aged much more slower than the beginning. So just the first $30 \%$ reductions were fit. At $70^{\circ} \mathrm{C}$ heating, the tiles were measured every 96 hours. There were still some micro-cracks on the BC-408 surface, these cracks were 1-2 $\mathrm{mm}^{-2}$ density. After 480 hours the light output changed in the characteristics of the tiles do not exceed $22-28 \%$. At $60^{\circ} \mathrm{C}$ heating, the tiles were measured every 180 hours. The tiles surfaces were no micro-cracks on the surface. After 540 hours the light output changed in the characteristics of the tiles do not exceed 21-26\%. There different temperature heating results all show that the tiles of BC-408 had less reduction than ND-100.

We define the operational time to be the time when the light output decreased by $20 \%$. The operational time at 0 degrees(the normal temperature at LHAASO site) can be determined from 
80 degree

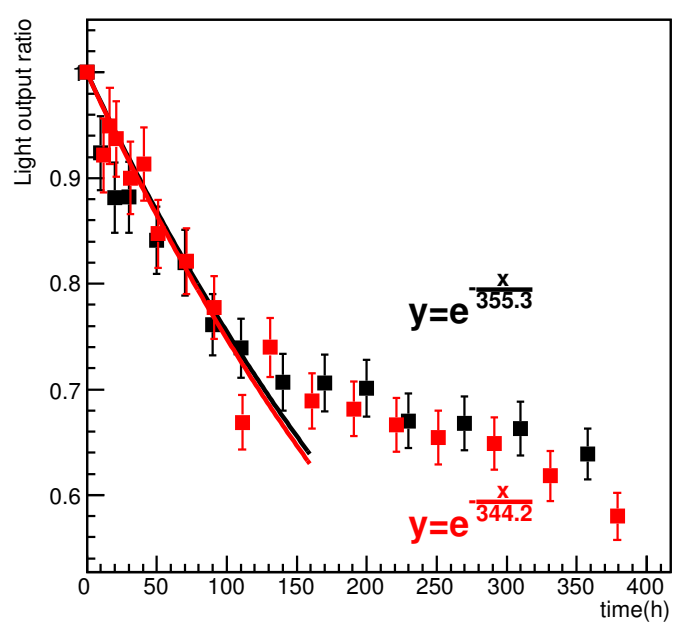

70 degree

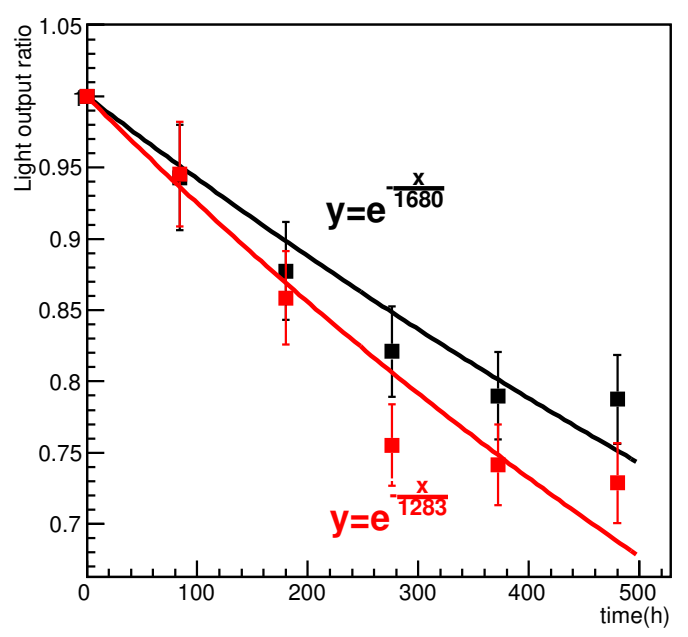

60 degree

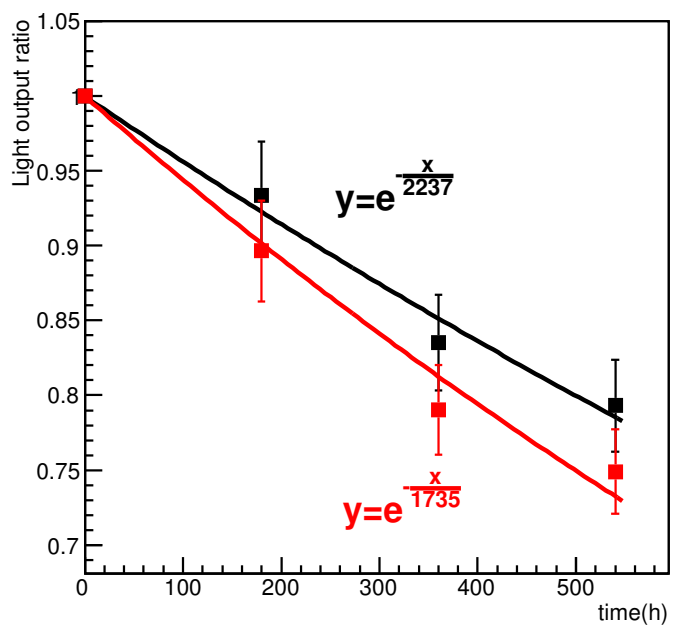

Figure 2: The light output ratio for different temperatures. The black dots are BC-408, the red ones are ND-100. 
Arrhenius plot:

$$
\ln (t)=\frac{A}{T}+B
$$

where $\mathrm{T}$ is thermodynamic temperature, $\mathrm{t}$ is the corresponding operational times, $\mathrm{A}$ and $\mathrm{B}$ are the coefficient related to the PS property. According to the result of accelerated aging at $60^{\circ} \mathrm{C}, 70^{\circ} \mathrm{C}$ and $80^{\circ} \mathrm{C}$, the Arrhenius plots for BC-408 and ND-100 are drawn in Figure 3 and Figure 4). The operational time predictions for a $20 \%$ change of light yield at 0 degree can be calculated by using Arrhenius plot. It can be seen the operational time at LHAASO site for scintillator BC-408 is 310.7 $\mathrm{yr}$ and for ND-100 is $62.8 \mathrm{yr}$.

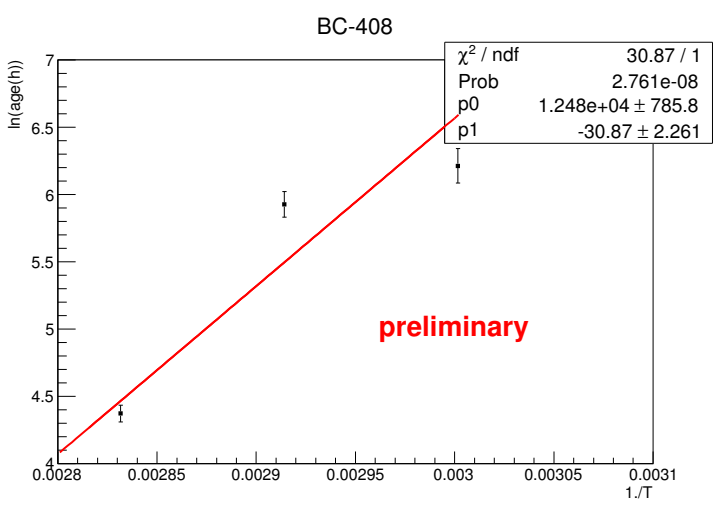

Figure 3: Arrhenius plot of BC-408.

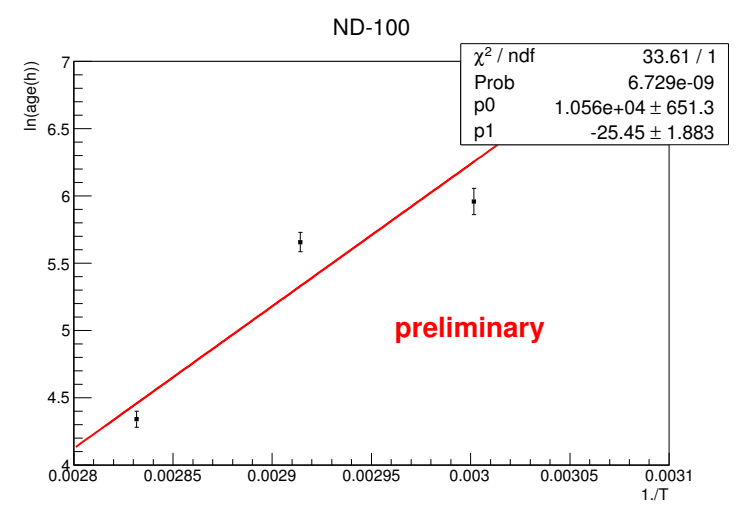

Figure 4: Arrhenius plot of ND-100.

\section{Conclusion}

It is important to study long-term stability of plastic scintillator for the LHAASO-KM2A. According to accelerated thermal aging test simulating the LHAASO site environment, it can be seen that the operational time BC-408 with WLS fiber readout is $310.7 \mathrm{yr}$ and ND-100 is $62.8 \mathrm{yr}$. Because of the experiment system errors is $2.2 \%$, the errors of the simulation age is about 3.4 times of simulation age which is the same as simulated. The simulation age errors will be reduced by using much more accurate measurement in the future. This work is supported by National Natural Science Foundation (NSFC) of China under contacts No. 11635011. 


\section{References}

[1] Cao Zhen, For the LHAASO Collaboration, Nucl. Instrum. Methods Phys. Res. A 742(2014) 95-98

[2] Shuwang Cui, For the LHAASO Collaboration, Astopart. Phys. 54(2014) 86íC92.

[3] Jia Liu,Proceedings of the 34th ICRC, PoS(ICRC2015)1007, The Hague,2015.

[4] Karpuchin, O.N., Plast. Massy. No 11, pp. 27-29,(1978)

[5] V. Senchishin et al., New Radiation Stable and Long-Lived Plastic Scintillator for the SSC. (1993)

[6] B.V. Grinyov et al. Radiation Measurements 38 (2004) 825 ÍC 828 